\title{
The Summary Loop: Learning to Write Abstractive Summaries Without Examples
}

\author{
Philippe Laban \\ UC Berkeley
}

\author{
Andrew Hsi \\ Bloomberg
}

\author{
John Canny \\ UC Berkeley
}

\author{
Marti A. Hearst \\ UC Berkeley*
}

\begin{abstract}
This work presents a new approach to unsupervised abstractive summarization based on maximizing a combination of coverage and fluency for a given length constraint. It introduces a novel method that encourages the inclusion of key terms from the original document into the summary: key terms are masked out of the original document and must be filled in by a coverage model using the current generated summary. A novel unsupervised training procedure leverages this coverage model along with a fluency model to generate and score summaries. When tested on popular news summarization datasets, the method outperforms previous unsupervised methods by more than 2 R-1 points, and approaches results of competitive supervised methods. Our model attains higher levels of abstraction with copied passages roughly two times shorter than prior work, and learns to compress and merge sentences without supervision.
\end{abstract}

\section{Introduction}

Summarization, or the task of condensing a document's main points into a shorter document, is important for many text domains, such as headlines for news and abstracts for research papers.

This paper presents a novel unsupervised abstractive summarization method that generates summaries directly from source documents, without the aid of example summaries. This approach simultaneously optimizes for the following important properties of a good summary:

- coverage of the keywords of the document,

- fluency of generated language, and

- brevity of generated summaries.

*Author emails: \{phillab,canny,hearst $\} @$ berkeley.edu, ahsil@bloomberg.net

\begin{abstract}
Original Document: Chilean President announced Wednesday that his country, which has been paralyzed by protests over the last two weeks, will no longer host two major international summits. [...] The President has now canceled the hosting of the economic APEC forum and COP25 environmental summit, which were both due to take place later this year. [...]
\end{abstract}

Masked Document: _-__ _-_- announced Wednesday that his country, which has been _-_- by _-_- over the last two weeks, will no longer ---- two major international _-_.. [...] The _-_- has now _-_- the --place later this ---.. [...]

Summary Loop [10 word constraint]: Pinera cancelled the APEC summit at Santiago.

Coverage Score: 0.22

Summary Loop [24 word constraint]: Pinera said Chileans have been canceled the hosting of the APEC summit, which was scheduled to take place in November. Coverage score: 0.33

Summary Loop [45 word constraint]: Sebastian Pinera announced Wednesday that his country will not hold the APEC summit, which was scheduled to take place in Santiago. Pinera said that Chileans had been paralyzed by protests over the last two weeks.

Coverage score: 0.39

Figure 1: Motivating example. A document from CNN.com (keywords generated by masking procedure are bolded), the masked version of the article, and generated summaries by three Summary Loop models under different length constraints.

One of the main contributions of this work is a novel method of inducing good coverage of important concepts from the original article. The coverage model we propose takes as input the original document with keywords masked out (see Figure 1). It uses the current best automatically generated summary to try to uncover the missing keywords. The more informative the current summary is, the more successful the coverage model is at guessing the blanked out keywords from the original document. A resulting coverage score is fed back into the training process of the summarization model 
with the objective of producing summaries with high coverage.

A second contribution is our unsupervised training procedure for summarization, the Summary Loop, which leverages the coverage model as well as a simple fluency model to generate and score summaries. During training, the procedure is conditioned on a desired summary length, forcing the Summarizer model to adapt to a length budget. Figure 1 shows Summary Loop summaries obtained for the same document under three different length budgets.

A third contribution is a set of specialized techniques employed during training to guide the model away from pathological behavior. These guard rails include a method for reducing repetition, for encouraging the model to complete sentences, and to avoid frame filling patterns.

The models trained through the Summary Loop outperform all prior unsupervised summarization methods by at least 2 ROUGE- 1 points on common news summarization datasets (CNN/DM and Newsroom), and achieve within a few points of state-of-the-art supervised algorithms, without ever being exposed to any summaries. In addition, summaries generated by our method use $50 \%$ more summarization techniques (compression, merging, etc.) than prior automatic work and achieve higher levels of abstraction, reducing by almost half the gap between human-generated summaries and automatic summaries in terms of length of copied spans.

\section{Related Work}

\section{Supervised Abstractive Summarization.} Sequence-to-sequence (seq2seq) (Sutskever et al., 2014) models trained using teacher-forcing are the most common approach to abstractive summarization (Nallapati et al., 2016). A common architecture is the Pointer-Generator (See et al., 2017). Performance can further be improved by constraining the attention (Gehrmann et al., 2018; Gui et al., 2019; Wang et al., 2019) and using pretrained Transformer-based language models (Lewis et al., 2019; Chi et al., 2019; Edunov et al., 2019). Through architectural changes, the training procedure remains constant: using a large corpus of document-summary pairs, the model is trained to reproduce target summaries.

Unsupervised Summarization. Most unsupervised summarization work is extractive: sentences deemed relevant are pulled out of the original document and stitched into a summary, based on a heuristic for a sentence's relevance (Mihalcea and Tarau, 2004; Barrios et al., 2015; West et al., 2019). Nikolov and Hahnloser (2019)'s abstractive approach is partially unsupervised, not requiring parallel data, but only a group of documents and a group of summaries. In contrast, our work does not require any summaries, and is trained using only documents. Radford et al. (2019) summarize documents using a language model (GPT2) in a Zeroshot learning setting. The model reads the document followed by a special token "TL/DR", and is tasked with continuing the document with a summary. Our work is an extension of this work: we initialize our Summarizer model with a GPT2 and specialize it with a second unsupervised method.

Summarization and Q\&A. Eyal et al. (2019) and Arumae and Liu (2018) turn reference summaries into fill-in-the-blank (FIB) questions, either as an evaluation metric or to train an extractive summarization model. In this work, we directly generate FIB questions on the document being summarized, bypassing the need for a reference summary.

Scialom et al. (2019)'s work stays closer to a Q\&A scenario, and uses a Question Generation module to generate actual questions about the document, answered by a Squad-based (Rajpurkar et al., 2018) model using the generated summary. We refrain from using actual questions because question generation remains a challenge, and it is unclear how many questions should be generated to assess the quality of a summary.

RL in Summarization. Paulus et al. (2018) introduced Reinforcement Learning (RL) to neural summarization methods by optimizing for ROUGE scores, leading to unreadable summaries. Since then, Reinforcement Learning has been used to select sentences with high ROUGE potential (Chen and Bansal, 2018), or optimize modified versions of ROUGE that account for readability (Pasunuru and Bansal, 2018). In all cases, the reward being computed relies on a reference summary, making the methods supervised. We craft a reward that does not require a target summary allowing our training process to remain unsupervised.

\section{The Summary Loop}

For this work, the definition of a summary is:

$$
\text { "A summary is a brief, fluent text that }
$$


covers the main points of an original document."

Brevity, fluency and coverage are the three pillars of a good summary. Under a length constraint, a good quality summary should contain as much information about the original document as possible while retaining fluent and coherent English.

Subsection 3.1 lays out the steps in the Summary Loop. Subsections 3.2-3.5 specify how each component is represented by a neural network. Section 4 shows how to train a summarizer model using this architecture in an unsupervised manner. ${ }^{1}$

\subsection{Summary Loop Steps}

Numbers in Figure 2 correspond to the following steps:

1. Summarizer receives a document $D$ and length-constraint L, and produces a summary $S$ fulfilling the length constraint.

2. Using a Masking Procedure, D is modified into a masked document $\mathrm{M}$, where important words have been replaced with blanks.

3. Coverage receives $\mathrm{S}$ and $\mathrm{M}$, and uses them to fill in each blank in $\mathrm{M}$ with a word, producing F. F and D are compared, and the resulting fill-in accuracy is called the Coverage Score.

4. Fluency receives $S$, and gives a Fluency Score based on its assessment of the quality of the Summary's writing.

5. The Fluency Score is added to the Coverage Score (as a weighed sum) into a Summary Score for the (D, S) pair.

6. Reinforcement Learning is used to train the Summarizer to produce summaries with high Summary Score.

The Summary Loop does not rely on the use of a target/reference/human-written summary, but only the summaries produced by the Summarizer model. The process can therefore be iterated upon without supervision from Summarization datasets.

\subsection{Summarization Model}

We use a Generative Transformer (Radford et al., 2019) as the model architecture of the summarizer. We make this choice for two reasons. First, Generative Transformers can produce text one word at a time, allowing the system to produce abstractive

\footnotetext{
${ }^{1}$ The code, model checkpoints and other resources are available at https://github.com/CannyLab/ summary_loop.
}

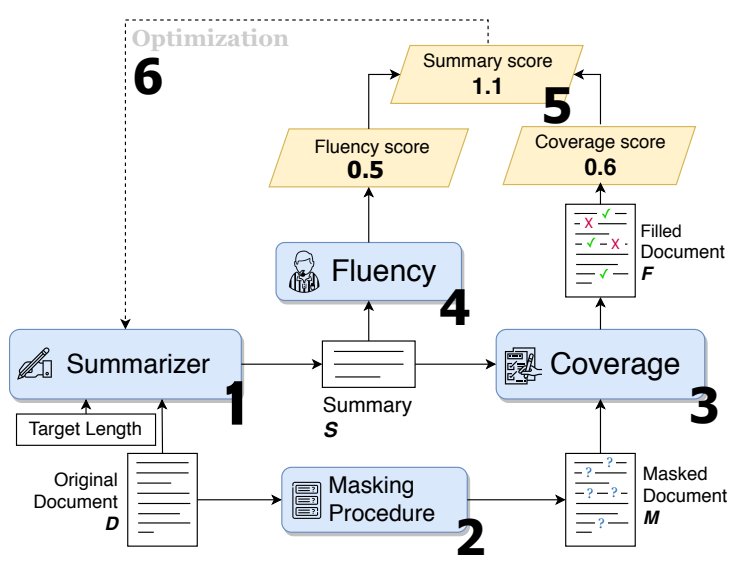

Figure 2: The Summary Loop involves three neural models: Summarizer, Coverage and Fluency. Given a document and a length constraint, the Summarizer writes a summary. Coverage receives the summary and a masked version of the document, and fills in each of the masks. Fluency assigns a writing quality score to the summary. The Summarizer model is trained, other models are pretrained and frozen.

summaries. Second, we use the pretrained Generative Transformer to initialize the Summarizer.

Practically, the Summarizer first reads through the entire document, followed by a special START token, signaling summarization. The Summarizer produces a probability distribution over words in its vocabulary, and a word is picked from the distribution and fed back as an input into the model. This procedure is repeated and halts either when the summary reaches a length constraint, or when the Summarizer produces a special END token. See Appendix $\mathrm{C}$ for the model size and initialization used to train the summarization paper.

\subsection{Masking Procedure}

The Masking Procedure decides on a set of keywords that are important elements in the document that should be recoverable using a summary. The keywords are replaced with blanks, indirectly indicating which information should be present in the summary. We use a tf-idf-based approach to decide on the set of masked keywords, as it is both simple and has been shown to represent word relevance to a document (Ramos, 2003). Masking procedure implementation details are presented in Section A of the Appendix.

We select the $\mathrm{k}$ words with highest tf-idf score for the document to serve as the masked words. The k parameter represents a balance: if too many words are masked, the filling-in becomes impos- 


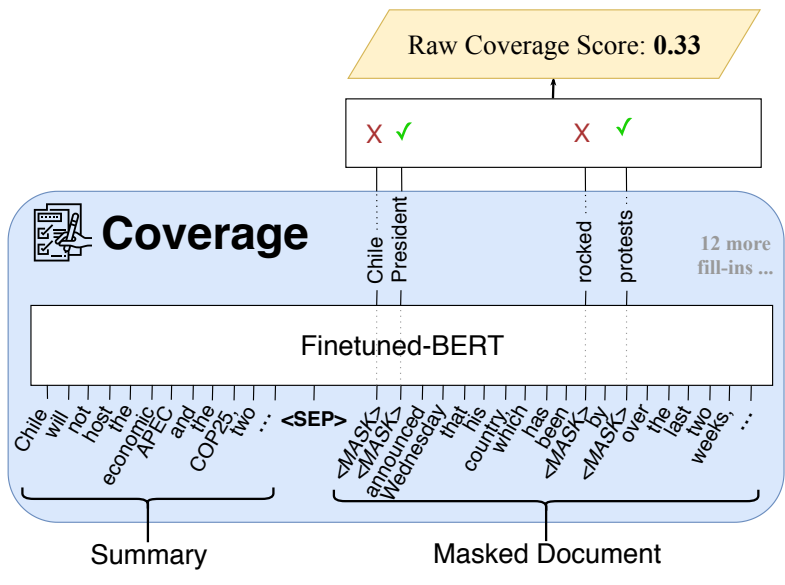

Figure 3: The Coverage model uses a finetuned BERT model. The summary is concatenated to the masked document as the input, and the model predicts the identity of each blank from the original document. The accuracy obtained is the raw coverage score.

sible, but if too few are masked, the Summarizer model will not be encouraged to include sufficient content in its summary. Varying the value of $k$ $(10,12,15,20)$ yielded only small discernible difference in the Summarizers produced, and we use $k=15$ in all our final experiments.

The masking procedure can be adapted to a specific domain. For instance, if summarizing financial documents, the masking procedure could systematically mask all numbers, encouraging the Summarizer model to add numbers to its summary.

\subsection{Coverage Model}

The Coverage Model receives a computationally generated summary and the masked document and attempts to fill in each blank word. The task of filling in blanks is similar to masked language modeling (MLM), used to pretrain BERT-like (Devlin et al., 2019) models. In MLM, some of the words are replaced with a special $M A S K$ token, and the model must use other information (unmasked words) to fill in the masked words. Because of the similarity to our task, we use a BERT-based neural network as the architecture for the coverage model. However, the coverage task differs from MLM in two ways. First, we modify the masking procedure: instead of masking a random percentage of the words (often $15 \%$ for BERT), we mask all appearances of the keywords selected by the masking procedure described in Section 3.3. Second, the input to the coverage model is a concatenation of the unmasked summary, a separator token and the masked document. The model can leverage un- masked information available in the summary to fill in the masked document. The Coverage Model is illustrated in Figure 3.

\subsubsection{Computing a Coverage Score}

Using the masking procedure, we obtain $M=$ $f(D)$, the masked document. The coverage model produces the filled document $F=g(M, S)$. Raw coverage score is the fraction of correctly filled in words in F. Let $D_{i}, F_{i}$ and $M_{i}$ correspond to the $i$ th word in their respective document, $I_{M}$ the set indices of words that have been masked. Then:

$$
\operatorname{Raw} \operatorname{Cov}(D, S)=\frac{\| i \in I_{M} \text { if } D_{i}=F_{i} \|}{\left\|I_{M}\right\|}
$$

The model can use information in the unmasked (visible) words of M to predict the masked words. For instance, if the word "Chile" is visible, then "Santiago" would be a well-informed guess near the word "capital", which might not be masked out. This is undesirable, because coverage should account for what information the model can learn from the summary S, not what it can guess from the unmasked portion of $\mathrm{D}$. To counteract this problem, we modify the raw coverage score by computing how much information the model can guess without the summary present, using an empty string summary: $F_{\emptyset}=g(M$, “"). We then normalize a summary's coverage by subtracting the empty string coverage from the raw coverage, leaving only filled-in words answerable using $\mathrm{S}$, as shown in Equation 2.

$$
\begin{aligned}
& \operatorname{Norm} \operatorname{Cov}(D, S)= \\
& \operatorname{Raw} \operatorname{Cov}(D, S)-\operatorname{Raw} \operatorname{Cov}(D, “ ”)
\end{aligned}
$$

In a nutshell, raw coverage score answers the question: "What fraction of blanked words can be correctly filled in with this summary?" and normalized coverage score answers: "What is the increase in the fraction of blanks that can be correctly filled in with this summary, compared to having no summary?" In the rest of this paper, Coverage Score refers to Normalized Coverage Score.

\subsubsection{Training the Coverage Model}

We train the Coverage Model once, and its weights are then fixed during the training of the Summarizer. In order to train the Coverage Model, we need pairs of documents (D) and summaries (S). However, we operate under the assumption that we do not have access to summaries (to keep the procedure unsupervised). In order to remove this dependency, we use the first 50 words of the unmasked 


\begin{tabular}{rccc}
$\begin{array}{r}\text { Summary } \\
\text { Dataset }\end{array}$ & $\begin{array}{c}\text { Summary } \\
\text { Length }\end{array}$ & $\begin{array}{c}\text { Raw } \\
\text { Coverage }\end{array}$ & $\begin{array}{c}\text { Norm. } \\
\text { Coverage }\end{array}$ \\
\hline Empty String & 0 & 0.334 & 0 \\
\hline Headline & 9.59 & 0.478 & 0.144 \\
First 10 words & 10.0 & 0.428 & 0.094 \\
\hline Newsroom & 23.41 & 0.525 & 0.191 \\
First 24 words & 24.0 & 0.537 & 0.203 \\
\hline CNN/DM & 45.75 & 0.726 & 0.392 \\
First 46 words & 46.0 & 0.649 & 0.315 \\
\hline
\end{tabular}

Table 1: Analysis of the raw and normalized coverage of three existing human-written summary datasets, as well as first-k word baselines.

document $(D[: 50])$ as a proxy for document summaries. The Coverage Model is initialized with a trained BERT model (Devlin et al., 2019), and trained using $(D, D[: 50])$ pairs on the coverage task. Because BERT is already trained on the similar MLM task, the Coverage model is able to leverage knowledge accrued by BERT. The Coverage Model converges after roughly 5 hours of training on a Titan X GPU.

\subsubsection{Analysis of Coverage}

We present properties of the raw and normalized coverage through the analysis of existing humanwritten summary datasets. We focus our analysis on three datasets in the news domain: (1) a headline dataset obtained from common US news websites (Laban and Hearst, 2017), (2) the Newsroom dataset (Grusky et al., 2018), and (3) the CNN/DM dataset (Nallapati et al., 2016).

For each dataset, we take document/summary pairs and obtain raw and normalized coverage score through our Coverage model, reported in Table 1.

First, longer summaries obtain higher coverage scores: a CNN/DM summary with an average of 45 words can be used to fill in $73 \%$ of the blanks correctly, compared to $48 \%$ for a 9 word headline. Across datasets, the correlation between summary length and raw coverage score is 0.56 , confirming that longer summaries contain more information, according to coverage.

Second, we simulate the first $\mathrm{k}$ words ${ }^{2}$ of the document as a summary. We use $k=10,24,46$ to match average word length in the three datasets. For two of the three values (10 and 46), the coverage of human-written summaries is higher than the first-k word counterpart. This is remarkable: even though the summary is farther away lexically (i.e.,

\footnotetext{
${ }^{2}$ We choose the first $\mathrm{k}$ words due to the similarity to Lede 3 (first 3 sentences), a common baseline in news.
}

is not a subset of the original words), it obtains higher coverage, demonstrating that the coverage model can account for reworded information.

\subsection{Fluency Model}

A model solely trained to optimize coverage has no incentive to write in good English, use punctuation, determinants or pronouns, as these are not words removed by the masking procedure. The objective of a Fluency Model is to judge the writing quality of the summary, independent of its coverage.

Given the right corpus, we argue that a language model's probability can be modified into a Fluency Score. Therefore, we adapt a language model into the Fluency Model.

We choose the generative Transformer (Radford et al., 2019) architecture for our Fluency model, as it can be trained into a powerful language model. Just as with the Summarizer, by using a standardized architecture and model size, we can make use of pretrained models. However, it is important for Fluency to fine tune the language model on the target domain, so that the Summarizer is rewarded for generating text similar to target content.

To produce a uniform Fluency Score, we linearly scale the language model's log-probability of a given summary $(L M(S))$ between an ideal value $L P_{l o w}$ and a maximum value $L P_{\text {high }}$ :

$$
\operatorname{Fluency}(S)=1-\frac{L M(S)-L P_{\text {low }}}{L P_{\text {high }}-L P_{\text {low }}}
$$

This ensures that the Fluency $(S)$ is usually in the range $[0,1] . L P_{\text {low }}$ and $L P_{\text {high }}$ are picked specifically for a particular language model, and ensure that the log-probability magnitudes of a specific language model do not affect the overall scores.

\subsection{Summary Score}

The final Summary Score is a weighed sum of the Coverage and Fluency Scores:

$$
\begin{aligned}
& \operatorname{SummaryScore}(D, S)= \\
& \alpha \cdot \operatorname{Norm} \operatorname{Cov}(D, S)+\beta \cdot \operatorname{Fluency}(S)
\end{aligned}
$$

$\alpha, \beta$ are hyperparameters giving relative importance to Coverage and Fluency. We set $\alpha=5$, $\beta=1$ in all our experiments. Model choice, size, and initialization are summarized in Figure A1.

\section{Training Procedure}

We first outline the training procedure and then detail several guard-rail mechanisms used during 
training to prevent the Summarizer from learning pathological writing strategies. Figure A2 presents training plots of a Summary Loop model and interpretation of the different learning phases.

\subsection{Training with Reinforcement Learning}

We use Reinforcement Learning to train the Summarizer component (agent), such that it achieves high summary score (reward). Note that the Coverage and Fluency models are frozen, and their weights are not trained. We make this choice as allowing Fluency and Coverage models to evolve could enable the models to coordinate and cheat.

We use the Self-critical sequence training (SCST) method (Rennie et al., 2017), as it has been shown to perform well on similar text generation tasks optimizing BLEU for image captioning or ROUGE scores in summarization.

In SCST, the Summarizer is used to produce two summaries of document $D$ : a greedy summary $\hat{S}$, using a decoding strategy that always picks the most likely next word, and a sampled summary $S^{s}$, picking the next word in the summary by sampling from the word distribution.

Summaries are scored using the Summary Loop:

$$
\begin{aligned}
\hat{R} & =\text { SummaryScore }(D, \hat{S}) \\
R^{s} & =\text { SummaryScore }\left(D, S^{s}\right)
\end{aligned}
$$

Then we minimize the following loss:

$$
L=\left(\hat{R}-R^{s}\right) \sum_{i=0}^{N} \log p\left(w_{i}^{s} \mid w_{1}^{s}, \ldots, w_{i-1}^{s}, D\right)
$$

Where $p\left(w_{i}^{s} \mid \ldots\right)$ represent the probability of the $i$ th word conditioned on previously generated word, according to the model.

Intuitively, if $R^{s}>\hat{R}$, minimizing $\mathrm{L}$ maximizes the likelihood of the sampled sequence - which is desired because it outperformed the greedy summary - and increases expected reward of the model.

\subsection{Training guard rails}

During training, the Summarizer model learns pathological summarization strategies. We build training guard rails to detect the pathological behavior and penalize the model during training.

A guard rail has a binary effect: if a pathology is detected in a summary, its Summary Score is reduced by a penalty amount $\delta$. We use $\delta=2$ for all experiments. We found three training guard rails to be useful: No-repetition, Finish-your-sentence, and No-frame-filling.

\subsubsection{No-repetition}

A common problem in neural text generation is repetition of text. Based on the observation that 3 -grams seldom repeat in common summarization datasets, the "No-repetition" training guard rail raises a penalty on a summary when it contains any repeated 3-gram.

\subsubsection{Finish-your-sentence}

When generating a summary, the model can either produce the END token, or generate a number of words up to the length constraint. We observe that if the model does not produce the END token, it often generates partial sentences, which is undesirable. Because we want to encourage the model to generate an END token, the "Finish-your-sentence" raises a penalty if a summary has no END token.

\subsubsection{No-frame-filling}

During training, the model sometimes learns to overly rely on sentence patterns that achieves high reward as a one size fits all summary. In one example the model learns to produce summaries solely of the form: " $\mathrm{X}$ talks with $\mathrm{Y}$ about the $\mathrm{Z}$ ". The model uses this frame, filling in the $\mathrm{X}, \mathrm{Y}$ and $\mathrm{Z}$ slots with relevant keywords and entities to achieve a small but positive coverage. This form of framefilling is undesirable, as the model often produces inaccurate information to fit the entities to the pattern.

We implement a guard rail to penalize the model when frame-filling patterns are observed. During training, we keep track of the last 100 summaries produced by the model. We then aggregate the frequency of words for each word position in the 100 summaries. If any word appears more than $50 \%$ of the time at a specific word position, we raise the "No-frame-filling" penalty. In the example given above, the word "talks" appeared in the second word position in more than $50 \%$ of the summaries, as well as the word "about" in the fifth position.

These rule-based training guard rails are simple and effective. In our finalized trained models, very few summaries exhibit penalized behavior: $2 \%$ for no-repetition, $5 \%$ for finish-your-sentence, and $2.5 \%$ for no-frame-filling.

\section{Results}

We present results for Summary Loop models trained in the news domain under three different length constraints: 10,24 , and 46 words, matching the distributions of the Headline, Newsroom 


\begin{tabular}{|c|c|c|c|c|c|c|}
\hline Method & R-1 & $\mathbf{R}-\mathbf{2}$ & $\mathbf{R}-\mathbf{L}$ & $\begin{array}{l}\text { Coverage } \\
\text { Score }\end{array}$ & $\begin{array}{l}\text { Fluency } \\
\text { Score }\end{array}$ & $\begin{array}{l}\text { Brevity } \\
\text { (avg words) }\end{array}$ \\
\hline \multicolumn{7}{|c|}{ Baselines } \\
\hline Human-written Summaries & 100 & 100 & 100 & 0.392 & 0.612 & 58.5 \\
\hline $\mathbb{X}$ Lead-3 baseline & 40.3 & 17.7 & 36.6 & 0.421 & 0.656 & 84.0 \\
\hline \multicolumn{7}{|c|}{ Supervised Methods } \\
\hline Pointer Generator (See et al., 2017) & 36.4 & 15.7 & 33.4 & 0.342 & 0.547 & 55.6 \\
\hline PG + Coverage (See et al., 2017) & 39.5 & 17.3 & 36.4 & 0.377 & 0.508 & 61.7 \\
\hline Bottom-Up (Gehrmann et al., 2018) & 41.2 & 18.7 & 38.3 & 0.378 & 0.538 & 73.9 \\
\hline$P E G A S U S_{B A S E}$ (Zhang et al., 2019a) & 41.8 & 18.8 & 38.9 & - & - & - \\
\hline PEGASU $S_{L A R G E}$ (Zhang et al., 2019a) & 44.1 & 21.3 & 40.9 & - & - & - \\
\hline \multicolumn{7}{|c|}{ Unsupervised Methods } \\
\hline $\mathbb{X}$ TextRank (Mihalcea and Tarau, 2004) & 35.2 & 12.9 & 28.7 & 0.370 & 0.612 & 49.62 \\
\hline GPT2 Zero-Shot (Radford et al., 2019) & 29.3 & 8.3 & 26.6 & - & - & - \\
\hline Summary Loop 45 & 37.7 & 14.8 & 34.7 & 0.404 & 0.627 & 47.0 \\
\hline
\end{tabular}

Table 2: ROUGE Results (F-1) on the non-anonymized CNN/DM test-set for supervised and unsupervised methods. Extractive methods indicated with $\mathbb{X}$. Our ROUGE scores have a 95\% confidence interval of at most \pm 0.30 . Coverage, Fluency and Brevity (average number of words) included for systems where summaries are available, using Coverage and Fluency models from our work.

\begin{tabular}{llll}
\hline Supervised Methods & R-1 & R-2 & R-L \\
\hline X Lead-3 baseline & $\mathbf{3 2 . 0}$ & $\mathbf{2 1 . 1}$ & $\mathbf{2 9 . 6}$ \\
PG + Coverage & 27.5 & 13.3 & 23.5 \\
\hline Unsupervised Methods & R-1 & R-2 & R-L \\
\hline X TextRank & 24.5 & $\mathbf{1 0 . 1}$ & 20.1 \\
Summary Loop 24 & $\mathbf{2 7 . 0}$ & 9.6 & $\mathbf{2 6 . 4}$ \\
\hline
\end{tabular}

Table 3: ROUGE Results on the released test set of Newsroom. X indicate extractive methods. Summary Loop outperforms other unsupervised method, is competitive with supervised Pointer-Generator.

(Grusky et al., 2018) and CNN/DM (Nallapati et al., 2016) datasets. We compare our summaries using the standard ROUGE metric, and by analyzing summaries for the errors made, the technique used and the level of abstraction. Finally, we show the Summary Loop can be complemented with supervision, reducing the amount of data needed to achieve comparable ROUGE results.

\subsection{News ROUGE Scores}

Table 2 and Table 3 present ROUGE results on the CNN/DM and Newsroom datasets respectively. In both cases, Summary Loop outperforms other unsupervised methods, and is competitive with supervised methods despite not being exposed to any example summaries. On CNN/DM, Summary Loop performs in between the Pointer Generator and Bottom Up architecture in terms of ROUGE-1. On the Newsroom, Summary Loop is within 0.6 ROUGE-
1 points of the Pointer-Generator with Coverage and surpasses it by 2 ROUGE-L points.

Recent breakthroughs in pretrained Transformer models have shown that using larger models in Summarization can lead to large improvements. For instance, a "large" version of the PEGASUS model (Zhang et al., 2019a) outperforms the "base" version by 2.3 ROUGE-1 points. Because Summary Loop experiments were performed using "base" models, we expect that using larger Transformer models could lead to similar gains.

Table 2 confirms that human-written summaries obtain amongst the highest Fluency and Coverage scores. Human-written summaries are only outperformed by Summary Loop summaries, and the Lede-3 baseline. However, the Summary Loop summaries are obtained by directly optimizing for Fluency and Coverage, and Lede- 3 baseline summaries achieve their higher Coverage at the expense of being much longer (i.e. 84 words on average compared to 58 in human-written summaries).

\subsection{Technique and Error Analysis}

We perform a manual analysis of 200 randomlyselected summaries on the test set of CNN/DM from the Pointer-Generator with Coverage (PGC), Bottom-Up (BU) and the unsupervised Summary Loop (SL). We annotated each summary with two types of errors: Inaccurate (information in summary contradicts document), Ungrammatical (one sentence or more is not properly constructed), and 


\begin{tabular}{lccc}
\hline Error Made & PGC & BU & SL \\
\hline Inaccurate (\%) & $\mathbf{1 1}$ & 31 & 24 \\
Ungrammatical (\%) & $\mathbf{7}$ & 15 & 18 \\
\hline Technique Used & PGC & BU & SL \\
(Success/Total) & $(\mathbf{S} / \mathbf{T})$ & $(\mathbf{S} / \mathbf{T})$ & $(\mathbf{S} / \mathbf{T})$ \\
\hline Sent. Compression & $86 / 110$ & $96 / 177$ & $118 / 194$ \\
Sent. Merging & $13 / 27$ & $29 / 65$ & $71 / 121$ \\
Novel Sentence & $0 / 1$ & $4 / 18$ & $33 / 70$ \\
Entity Manipulation & $7 / 10$ & $15 / 27$ & $27 / 40$ \\
Total Technique & $106 / 148$ & $144 / 287$ & $249 / 425$ \\
\hline
\end{tabular}

Table 4: Error and Technique analysis on 200 randomly selected summaries on the CNN/DM test-set for the Point-Gen with Cov. (PGC), Bottom-Up (BU) and unsupervised Summary Loop (SL). For each summarization technique, we report two numbers: the number of successful occurrences in summaries with no error, and the total number of occurrences in the 200 summaries.

four summarization techniques: Sentence Compression (summary sentence is a document sentence with words removed), Sentence Merging (2 or more document sentences are merged into a summary sentence), Novel Sentence (original sentence in the summary), and Entity Manipulation (a named entity is modified or simplified, e.g. changing a full name to a last name). We present Summary Loop examples illustrating each error and technique in Figures A3 - A8.

The analysis was performed by the first author of the paper, labeling article/summary pairs without knowledge of model origin. A summary can manifest any number of summarization Techniques, or none. Labeling is binary: if a summary exhibits more than one or instances of a Technique, it receives a 1 , otherwise it receives a 0 . Results of the analysis are summarized in Table 4.

SL uses significantly more summarization techniques (425) than PGC (148) and BU (287) summaries. Beyond raw counts, SL is more successful at applying summarization techniques (59\% success) than BU (50\% success), but less successful than PGC (72\%). Note however that PGC takes little risk: $19 \%$ of the summaries go beyond sentence compression, and $39 \%$ are extractive, using none of the summarization techniques.

\subsection{Level of Abstraction}

All methods generating summaries one word at a time have potential for abstraction. In Figure 4 we analyze human and system written summaries for abstraction level. We measure a summary's level of abstraction by looking at the length of spans

\begin{tabular}{|l|l|l|l|l|}
\hline Span & Gold & $\begin{array}{l}\text { Summary } \\
\text { Loop }\end{array}$ & $\begin{array}{l}\text { Bottom } \\
\text { Up }\end{array}$ & PG + Cov \\
\hline Novel & $9.8 \%$ & $\mid 0.6 \%$ & $1.3 \%$ & $\mid 1.0 \%$ \\
Length 1 & $23.6 \%$ & $6.0 \%$ & $\mid 2.9 \%$ & $1.4 \%$ \\
Length 2 & $20.8 \%$ & $11.4 \%$ & $\mid 4.5 \%$ & $\mid 1.1 \%$ \\
Length 3-5 & $24.7 \%$ & $26.4 \%$ & $13.0 \%$ & $3.6 \%$ \\
Length 6-10 & $12.0 \%$ & $29.7 \%$ & $21.1 \%$ & $9.3 \%$ \\
Length 11+ & $9.1 \%$ & $25.9 \%$ & $57.2 \%$ & $83.6 \%$ \\
\hline Avg. Length & 4.2 & 7.8 & 14.8 & 25.2 \\
\hline
\end{tabular}

Figure 4: Histogram and average copied span lengths for abstractive summaries. A summary is composed of novel words and word spans of various lengths copied from the document. Summary Loop summaries copy shorter spans than prior automatic systems, but do not reach abstraction levels of human-written summaries.

\begin{tabular}{lllll}
\hline Initialization Method & \multicolumn{1}{c}{$\mathrm{R}-1$} & $\mathrm{R}-2$ & $\mathrm{R}-\mathrm{L}$ & Test Loss \\
\hline \multicolumn{5}{c}{ 28k samples from CNN/DM $(10 \%)$} \\
\hline Random Initialization & 7.0 & 0.9 & 8.8 & 6.05 \\
GPT2 & 37.1 & 15.9 & 31.9 & 2.21 \\
Summary Loop S10 & $\mathbf{3 8 . 7}$ & $\mathbf{1 6 . 2}$ & $\mathbf{3 5 . 1}$ & 2.07 \\
\hline \multicolumn{5}{c}{ All of CNN/DN $(100 \%)$} \\
\hline Random Weights & 20.4 & 4.1 & 19.1 & 4.22 \\
GPT2 & 38.4 & 17.2 & 35.0 & 2.02 \\
Summary Loop S100 & $\mathbf{4 1 . 0}$ & $\mathbf{1 8 . 1}$ & $\mathbf{3 7 . 3}$ & 1.89 \\
\hline
\end{tabular}

Table 5: ROUGE Results on the CNN/DM test-set for supervised generative Transformers. Initializing with the unsupervised Summary Loop outperforms random and GPT2 initializations.

copied from the document. Summary Loop is the most abstractive automated method, although less so than human written summaries. SL cuts nearly in half the length of copied spans compared to other automated methods.

\subsection{Supervision is not the enemy}

If summaries are available, we show that they can complement the unsupervised Summary Loop. We run supervised experiments on CNN/DM using a generative Transformer architecture and varying the initialization. We compare initializing with (1) random weights, (2) the original GPT2 weights, and (3) the Summary Loop weights of target length 45. We train each model with teacher forcing, comparing using the entire CNN/DM training set to just $10 \%$ of it. The results are summarized in Table 5.

First, initializing with the Summary Loop leads to higher ROUGE score both in the $10 \%$ and full dataset setting. As expected, results improve when using the entirety of the data, and the Summary Loop initialized model trained with the entirety of CNN/DM obtains a ROUGE-1 F1-score of 41.0, 
within the confidence interval of the supervised Bottom Up (Gehrmann et al., 2018) architecture. This is a strong result as the Transformer we use is a generic language model, and is not specialized for summarization.

Second, initializing with Summary Loop and training with $10 \%$ of CNN/DM yields comparable ROUGE scores to initializing with GPT2 and using the entire CNN/DM, showing that Summary Loop can be useful when fewer summaries are available.

\section{Discussion}

Customizing summaries. In Figure 1, we illustrate the effect of the length constraint by summarizing the same document under three different length constraints. Each model adapts to its word budget. However, length is only one way to customize summaries. One might want to summarize based on point of view, chronology, theme, etc.

Fluency vs. Grammaticality. By choosing to represent the validity of summaries with a Language model, we encourage fluent summaries (i.e., with likely sequences of words) but not necessarily grammatical ones. Extending the scoring to include grammaticality, either by using a parsing model, or leveraging the Corpus of Linguistic Acceptability (Warstadt et al., 2019) could prove useful.

Summarization in the wild. Because our method is unsupervised, it can be applied to new domains and languages. In this work, we benefited from pretrained BERT and GPT2 models in English, which do not yet exist publicly for other languages. Once they become available in other languages, the Summary Loop can be ported over.

Abstraction dangers. Recent work around measuring factuality in generated text, using Natural Language Inference (Guo et al., 2018) or rule-based fact extraction (Zhang et al., 2019b) becomes increasingly important with summaries that are more abstractive. This work can be naturally included into the Summary Loop, with a fact-checker model generating an accuracy score.

\section{Conclusion}

In this work we present a new approach to unsupervised abstractive summarization based on maximizing a combination of coverage and fluency for a given length constraint. When tested on common news summarization datasets, our method significantly outperforms previous unsupervised methods, and gets within the range of competitive supervised methods. Our models attain levels of abstraction closer to human-written summaries, although with more abstraction, more potential for factual inaccuracies arise.

\section{Acknowledgments}

We would like to thank Forrest Huang, David Chan, Roshan Rao, Katie Stasaski and the ACL reviewers for their helpful comments. This work was supported by the first author's internship at Bloomberg, and a Bloomberg Data Science grant. We also gratefully acknowledge support received from an Amazon Web Services Machine Learning Research Award and an NVIDIA Corporation GPU grant.

\section{References}

Kristjan Arumae and Fei Liu. 2018. Reinforced extractive summarization with question-focused rewards. In Proceedings of ACL 2018, Student Research Workshop, pages 105-111.

Federico Barrios, Federico López, Luis Argerich, and Rosita Wachenchauzer. 2015. Variations of the similarity function of textrank for automated summarization. In Argentine Symposium on Artificial Intelligence (ASAI 2015)-JAIIO 44 (Rosario, 2015).

Yen-Chun Chen and Mohit Bansal. 2018. Fast abstractive summarization with reinforce-selected sentence rewriting. In Proceedings of the 56th Annual Meeting of the Association for Computational Linguistics (Volume 1: Long Papers), pages 675-686.

Zewen Chi, Li Dong, Furu Wei, Wenhui Wang, XianLing Mao, and Heyan Huang. 2019. Cross-lingual natural language generation via pre-training. arXiv preprint arXiv:1909.10481.

Jacob Devlin, Ming-Wei Chang, Kenton Lee, and Kristina Toutanova. 2019. Bert: Pre-training of deep bidirectional transformers for language understanding. In Proceedings of the 2019 Conference of the North American Chapter of the Association for Computational Linguistics: Human Language Technologies, Volume 1 (Long and Short Papers), pages 4171-4186.

Sergey Edunov, Alexei Baevski, and Michael Auli. 2019. Pre-trained language model representations for language generation. In Proceedings of the 2019 Conference of the North American Chapter of the Association for Computational Linguistics: Human Language Technologies, Volume 1 (Long and Short Papers), pages 4052-4059.

Matan Eyal, Tal Baumel, and Michael Elhadad. 2019. Question answering as an automatic evaluation metric for news article summarization. In Proceedings of the 2019 Conference of the North American 
Chapter of the Association for Computational Linguistics: Human Language Technologies, Volume 1 (Long and Short Papers), pages 3938-3948.

Sebastian Gehrmann, Yuntian Deng, and Alexander Rush. 2018. Bottom-up abstractive summarization. In Proceedings of the 2018 Conference on Empirical Methods in Natural Language Processing, pages 4098-4109.

Max Grusky, Mor Naaman, and Yoav Artzi. 2018. Newsroom: A dataset of 1.3 million summaries with diverse extractive strategies. In Proceedings of the 2018 Conference of the North American Chapter of the Association for Computational Linguistics: $\mathrm{Hu}$ man Language Technologies, Volume 1 (Long Papers), pages 708-719.

Min Gui, Junfeng Tian, Rui Wang, and Zhenglu Yang. 2019. Attention optimization for abstractive document summarization. In Proceedings of the 2019 Conference on Empirical Methods in Natural Language Processing and the 9th International Joint Conference on Natural Language Processing (EMNLP-IJCNLP), pages 1222-1228.

Han Guo, Ramakanth Pasunuru, and Mohit Bansal. 2018. Soft layer-specific multi-task summarization with entailment and question generation. In Proceedings of the 56th Annual Meeting of the Association for Computational Linguistics (Volume 1: Long Papers), pages 687-697.

Philippe Laban and Marti A Hearst. 2017. newslens: building and visualizing long-ranging news stories. In Proceedings of the Events and Stories in the News Workshop, pages 1-9.

Mike Lewis, Yinhan Liu, Naman Goyal, Marjan Ghazvininejad, Abdelrahman Mohamed, Omer Levy, Ves Stoyanov, and Luke Zettlemoyer. 2019. Bart: Denoising sequence-to-sequence pre-training for natural language generation, translation, and comprehension. arXiv preprint arXiv:1910.13461.

Rada Mihalcea and Paul Tarau. 2004. Textrank: Bringing order into text. In Proceedings of the 2004 conference on empirical methods in natural language processing, pages 404-411.

Ramesh Nallapati, Bowen Zhou, Cicero dos Santos, Ça glar Gulçehre, and Bing Xiang. 2016. Abstractive text summarization using sequence-to-sequence rnns and beyond. CoNLL 2016, page 280.

Nikola I Nikolov and Richard HR Hahnloser. 2019. Abstractive document summarization without parallel data. arXiv preprint arXiv:1907.12951.

Ramakanth Pasunuru and Mohit Bansal. 2018. Multireward reinforced summarization with saliency and entailment. In Proceedings of the 2018 Conference of the North American Chapter of the Association for Computational Linguistics: Human Language Technologies, Volume 2 (Short Papers), pages 646653.
Romain Paulus, Caiming Xiong, and Richard Socher. 2018. A deep reinforced model for abstractive summarization. In Proceedings of ICLR.

F. Pedregosa, G. Varoquaux, A. Gramfort, V. Michel, B. Thirion, O. Grisel, M. Blondel, P. Prettenhofer, R. Weiss, V. Dubourg, J. Vanderplas, A. Passos, D. Cournapeau, M. Brucher, M. Perrot, and E. Duchesnay. 2011. Scikit-learn: Machine learning in Python. Journal of Machine Learning Research, 12:2825-2830.

Alec Radford, Jeffrey Wu, Rewon Child, David Luan, Dario Amodei, and Ilya Sutskever. 2019. Language models are unsupervised multitask learners.

Pranav Rajpurkar, Robin Jia, and Percy Liang. 2018. Know what you dont know: Unanswerable questions for squad. In Proceedings of the 56th Annual Meeting of the Association for Computational Linguistics (Volume 2: Short Papers), pages 784-789.

Juan Enrique Ramos. 2003. Using tf-idf to determine word relevance in document queries.

Steven J Rennie, Etienne Marcheret, Youssef Mroueh, Jerret Ross, and Vaibhava Goel. 2017. Self-critical sequence training for image captioning. In Proceedings of the IEEE Conference on Computer Vision and Pattern Recognition, pages 7008-7024.

Thomas Scialom, Sylvain Lamprier, Benjamin Piwowarski, and Jacopo Staiano. 2019. Answers unite! unsupervised metrics for reinforced summarization models. In Proceedings of the 2019 Conference on Empirical Methods in Natural Language Processing and the 9th International Joint Conference on Natural Language Processing (EMNLP. IJCNLP), pages 3237-3247.

Abigail See, Peter J Liu, and Christopher D Manning. 2017. Get to the point: Summarization with pointergenerator networks. In Proceedings of the 55th Annual Meeting of the Association for Computational Linguistics (Volume 1: Long Papers), volume 1, pages 1073-1083.

Ilya Sutskever, Oriol Vinyals, and Quoc V Le. 2014. Sequence to sequence learning with neural networks. In Advances in neural information processing systems, pages 3104-3112.

Wenbo Wang, Yang Gao, He-Yan Huang, and Yuxiang Zhou. 2019. Concept pointer network for abstractive summarization. In Proceedings of the 2019 Conference on Empirical Methods in Natural Language Processing and the 9th International Joint Conference on Natural Language Processing (EMNLPIJCNLP), pages 3067-3076.

Alex Warstadt, Amanpreet Singh, and Samuel R Bowman. 2019. Neural network acceptability judgments. Transactions of the Association for Computational Linguistics, 7:625-641. 
Peter West, Ari Holtzman, Jan Buys, and Yejin Choi. 2019. Bottlesum: Unsupervised and self-supervised sentence summarization using the information bottleneck principle. In Proceedings of the 2019 Conference on Empirical Methods in Natural Language Processing and the 9th International Joint Conference on Natural Language Processing (EMNLPIJCNLP), pages 3743-3752.

Jingqing Zhang, Yao Zhao, Mohammad Saleh, and Peter J Liu. 2019a. Pegasus: Pre-training with extracted gap-sentences for abstractive summarization. arXiv preprint arXiv:1912.08777.

Yuhao Zhang, Derek Merck, Emily Bao Tsai, Christopher D Manning, and Curtis P Langlotz. 2019b. Optimizing the factual correctness of a summary: A study of summarizing radiology reports. arXiv preprint arXiv:1911.02541. 


\section{A Masking Procedure Details}

The masking procedure follows these steps:

1. We randomly sample 5,000 documents in the domain being summarized (e.g. News) as a training corpus,

2. The training corpus is tokenized using the tokenizer of the Coverage model. In our case, we tokenize with the Word Piece model of the BERT Base model (Devlin et al., 2019),

3. We train a tf-idf transformation model using the tokenized training corpus using default parameters of scikit-learn's tf-idf implementation (Pedregosa et al., 2011),

4. Given a document to be masked, we use the trained tf-idf model to produce a tf-idf for the document,

5. The words present in the document are ranked in decreasing order of tf-idf score, and the $k$ words with highest tf-idf form the masking set,

6. All occurrences of the words in the masking set are replaced by a mask in the document, creating the masked document.

\section{B Fluency Examples}

Table A1 provides examples from the Headline dataset of sampled headlines and their corresponding Fluency Score. The Fluency Score, a normalized language model log-perplexity, ranges from 0 to 1 . Even though all these headlines are written by a human, the Fluency scores vary, with the higherscoring headlines using more standard grammatical constructs. Note that the use of complex entity names does not prevent the model from obtaining a high Fluency score.

\begin{tabular}{lc}
\hline Example Headline & Fluency Score \\
\hline $\begin{array}{l}\text { Henry's Monaco recruit giant Brazil- } \\
\text { ian Naldo for relegation scrap }\end{array}$ & 0.16 \\
$\begin{array}{l}\text { Tesla shares dive after price cut, pro- } \\
\text { duction numbers }\end{array}$ & 0.41 \\
$\begin{array}{l}\text { French police arrest gilets jaunes } \\
\text { protests leader Eric Drouet }\end{array}$ & 0.59 \\
$\begin{array}{l}\text { Carlos Ghosn will appear in public for } \\
\text { the first time since his arrest }\end{array}$ & 0.75 \\
\hline
\end{tabular}

Table A1: Example selected headlines and their Fluency score. The headlines were picked from a corpus of human-written news headlines. The average Fluency in the corpus is 0.479 .

\section{Model Size and Initialization}

Figure A1 shows the model size and initialization model used for each of the Summarizer, Coverage and Fluency models.

Summarizer Architecture
GPT2-base: 12-layer, 768-hidden, 12-heads
Summarizer Initialization
GPT2 base model from Radford et al. (2019)
Coverage Architecture
BERT-base: 12-layer, 768-hidden, 12-heads
Coverage Initialization
Pretrained model obtained in Section 3.4.2
Fluency Architecture
GPT2-base: 12-layer, 768-hidden, 12-heads
Fluency Initialization
GPT2 base model from (Radford et al., 2019),
finetuned with Language modeling on news
text.

Figure A1: The model size choice as well as initialization method for the Summarizer, Coverage and Fluency models in the Summary Loop. Each model leverages a pretrained Transformer.

\section{Training Plots}

Figure A2 presents the plots of key variables we obtain during the training of the length 10 Summary Loop model. The training occurred over 10 days using a single Titan X GPU. During a first phase which occurs in the first 2 days of training, the model learns to copy content from the news article, which helps it achieve high Fluency and Coverage. In a second phase starting around the second day, the Summarizer learns to gain Coverage which maintaining Fluency mostly constant, which makes the overall Summary Score rise. The Summarizer model quickly learns to use its word budget, and after 10 days of training, the model uses an average of 9.7 words in its summaries.

\section{E Example Annotated Summaries}

Figures A3, A4, A5, A6, A7, and A8 show example documents and the generated Summary Loop summary from the error and technique analysis of Section 5.2. Each summary manifests a summarization technique or error observed. 


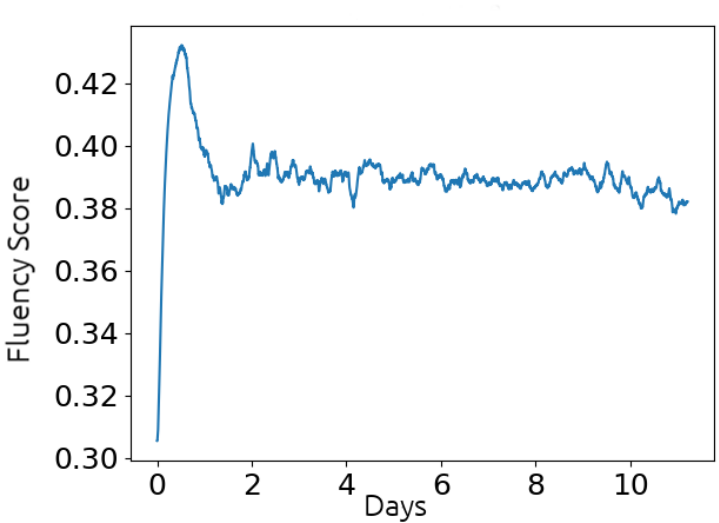

(a) Fluency Score

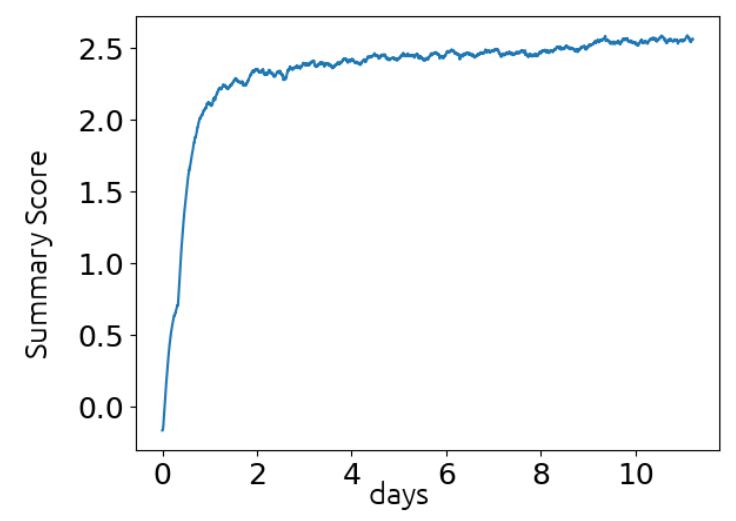

(c) Summary Score

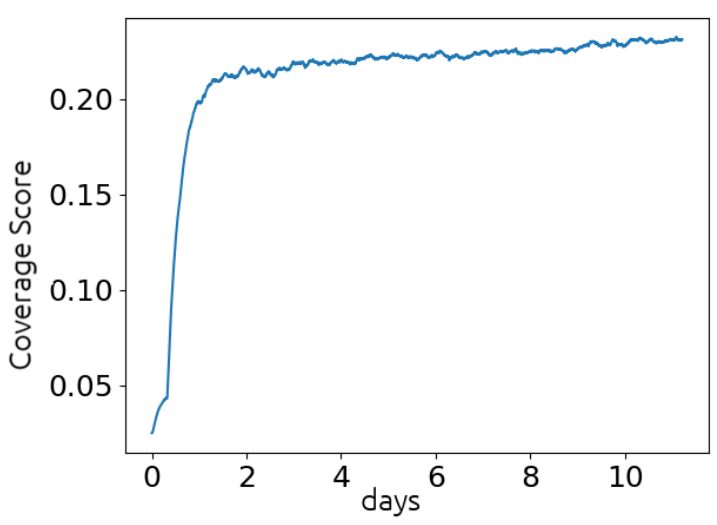

(b) Coverage Score

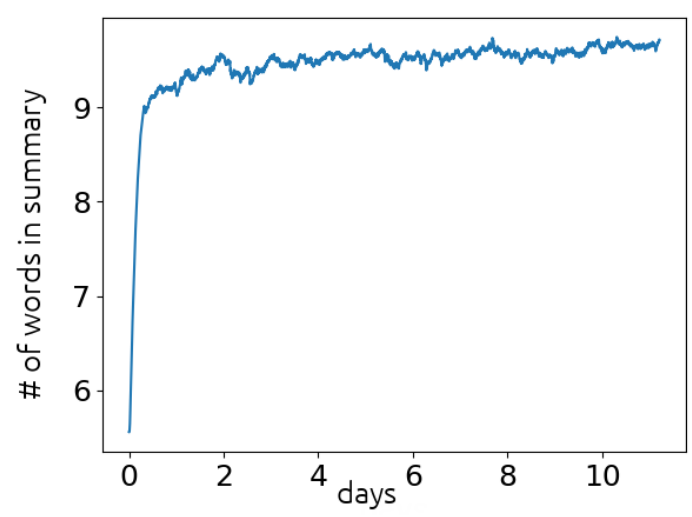

(d) Average number of words in summary

Figure A2: Plots of key variables during the training of the length 10 Summary Loop: (a) is a plot of the average Fluency Score, (b) is a plot of the average normalized Coverage Score, (c) is a plot of the average Summary Score (taking guard-rails into account), and (d) is a plot of the average number of words in summaries produced.

\section{Sentence Compression Example}

Document: He has long struggled to convince voters that he is a suitable choice for prime minister. Now Ed Miliband has hired a leadership coaching firm that helps people overcome anxiety and find their "inner voice". The consultants drafted in by the Labour leader claim to work with politicians to build "leadership skills" using "neuroscience" and "business psychology". Ed Miliband, pictured, has hired a US guru who can help him convince himself that he can be Prime Minister. [...]

Summary: Ed Miliband has hired a US guru who can help politicians on their leadership skills using neuroscience. Mr Miliband has hired the firm that can help politicians to build their leadership skills. The consultants drafted in by the Labour leader claim to work with politicians.

Figure A3: Summary Loop summary from the Error and Technique analysis (Section 5.2) illustrating the Sentence Compression technique. The blue boldface highlight is an example of sentence compression. 


\section{Sentence Merging Example}

Document: A single mom and her three kids who "lost everything but their lives" in the East Village apartment explosion last week are getting an incredible outpouring of support from their fellow New Yorkers. [...] Dr McLean, a 58-year-old child psychiatrist in the South Bronx, says she and daughter Rose, 8, and twins James and Annabelle, 5, had nothing more than the clothes on their backs after the disaster. Diane McLean, 58, and her three children lost "everything but their lives" when fire destroyed their apartment last week. Rose, 8, ( left ) and twins James and Annabelle, 5 , lost everything except the clothes on their backs in the fire that destroyed their apartment building. [..] A GoFundMe campaign has raised nearly $\$ 90,000$. [...]

Summary: Diane McLean says she and daughter Rose, 8, and twins James and Annabelle, lost everything but their lives at East Village apartment explosion last week. Diane McLean and her three kids had the clothes on their backs. A GoFundMe campaign has raised nearly \$90,000.

Figure A4: Summary Loop summary from the Error and Technique analysis (Section 5.2) illustrating the Sentence Merging technique. The bold blue and italicized red selections are two examples of sentence merging. In the blue example "Dr McLean" is replaced by "Diane McLean" in the summary, an example of entity manipulation.

\section{Novel Sentence Example}

Document: For most of us, the dream of a holiday home is one that will probably never be realised. But for the lucky minority with a few extra million in the bank, its seems the world is quite literally your oyster when looking for property around the world. From a Lake Garda mansion with a pool overlooking the water to an Italian villa that looks like a castle and an Antigua retreat with Giorgio Armani as a neighbour, these are some of the most spectacular holiday homes on the market at the moment. On the Lombardy side of Lake Garda, this Lionard property is a luxurious villa with one serious waterfront view. Lake Garda. On the Lombardy side of Lake Garda, in northern Italy, lies a luxury villa with a view - just several miles north of Brescia. And for $€ 18$ million ( about $£ 13$ million or $\$ 20$ million ) it can all be yours. Not only is there a large swimming pool looking out on the water, but also a large deck with plenty of space for sun beds, gazebos and al fresco dining spots, overlooking a 4000 square metre garden. Inside, the house is just as breathtaking. For about 18 million Euros ( or $\$ 13$ million ), the modern home, complete with pool, gazebo, and al fresco dining options, can be yours. [...]

Summary: The Lake Garda home is a luxury villa with a view on the Lombardy side of Lake Garda. This villa with gazebo and al fresco dining options. Inside, the house is just as breathtaking. For about 18 million Euros.

Figure A5: Summary Loop summary from the Error and Technique analysis (Section 5.2) illustrating the Novel Sentence technique. The first sentence of the summary uses pieces from the original document (in boldface blue) to form a sentence with an alternative but correct meaning. 


\section{Entity Manipulation Example}

Document: Sipping a glass of glorious red wine which has been carefully aged in a hand-crafted oak barrel is my idea of heaven. [...] A $\$ 5$ bottle has suddenly become $\$ 12$ because the wine has lingered in an oak barrel before bottling. So when I read this week about a new gadget that claims to be able to "oak age" wine in hours rather than years, my curiosity was seriously roused. The Oak Bottle promises to impart an authentic aged flavour - a process that can take up to two years - in just a day or two. Who wouldn't drink to that? Scroll down for video. TV wine expert Oz Clarke puts to the test this oak bottle that claims to "oak age" wine in hours rather than years. The product, which retails at $\$ 50$, is the brainchild of 30-year-old entrepreneur Joel Paglione. [...]

Summary: Joel Paglione said the Oak Bottle promises to be able to oak age wine in hours rather than years. The Oak Bottle promises an authentic aged flavour that can take up to two years. A bottle has been made in an oak barrel.

Figure A6: Summary Loop summary from the Error and Technique analysis (Section 5.2) illustrating the Entity Manipulation technique. The entity Joel Paglione (in boldface blue) is correctly inserted to represent the company.

\section{Inaccurate Example}

Document: The traditional cookie cutter wedding no longer exists - new reports suggest Brits are ditching tradition in favour of alternative practices when it comes to getting hitched. Two of the biggest changes are the fact that religious services have fallen out of favour and that brides are opting for bold colour schemes for their big day. A new study, which has tracked the decisions of brides and grooms over the past five years interviewed 1,893 newlyweds and compared them to answers they have collated since 2010. Scroll down for video. [...]

Summary: The new study showed that British couples are opting for religious ceremonies when it comes to their big day with services falling from 40 per cent of the past five years. The study showed that couples are opting to holiday in the UK.

Figure A7: Summary Loop summary from the Error and Technique analysis (Section 5.2) illustrating the Inaccurate error. The summary inaccurately claims religious ceremonies are increasing, when the document says they are in decline. Key phrases are highlighted in boldface blue. 


\section{Ungrammatical Example}

Document: Despite his daughter remaining in a medically induced coma since she was found unresponsive in a bathtub at her Atlanta home in January, singer Bobby Brown told an audience on Saturday night that she is "awake.". Bobby was performing at the Verizon Theatre in Dallas when he told the stunned audience that "Bobbi is awake. She's watching me." The singer didn't elaborate on if his daughter had regained consciousness or if he was talking instead about her spirit. After the 46-year-old's comment, his sister Tina posted on Facebook," [...] Whitney Houston's family insists the 22-year-old is not awake and is the same condition she was when she entered the facility. "She's in the exact same condition she was in when she went into the facility." a source told the site [...]

Summary: Bobby Brown was performing at the Verizon Theatre in Dallas when Bobbi was awake. He said that Tina posted on Facebook that her daughter was awake. She was the singer. She was going to be awake. She is the same condition.

Figure A8: Summary Loop summary from the Error and Technique analysis (Section 5.2) illustrating the Ungrammatical error. The last short summary sentence (in boldface blue) is not properly constructed, based on an unsuccessful attempt to compress a sentence in the document (also in boldface blue). 